\title{
Multilayered Waveguides for Increasing the Gain Bandwidth of Integrated Amplifiers
}

\author{
Athanasios Laliotis and Eric M. Yeatman, Senior Member, IEEE
}

\begin{abstract}
In this paper, we propose and demonstrate the use of multilayered (laminated) waveguides for increasing the available gain spectrum of sol-gel amplifiers. Experimental investigations of the effects of phosphorus codoping on the amplifier spectral characteristics and fluorescence decay are also presented. We demonstrate that the shape of the erbium emission cross section, for aluminophosphosilicate amplifiers, has a minor dependence on the amount of phosphorus doping and that spectral benefits are only achieved for aluminosilicate amplifiers, albeit with low levels of erbium inversion. Laminated amplifiers, consisting of alternating layers of two compositions, are shown to display novel spectral characteristics that cannot be obtained by the use of single composition cores. Internal gain, which is demonstrated for this device, also suggests that erbium inversion can be maintained at relatively high levels. Experimental results are supported by numerical analysis, and a 20-nm bandwidth increase is observed for laminated amplifiers, with gain levels up to $1.4 \mathrm{~dB} / \mathrm{cm}$ indicated, when processing conditions are optimized.
\end{abstract}

Index Terms-Er-doping, optical amplifier, sol-gel, thin films.

\section{INTRODUCTION}

$\mathbf{S}$ ILICA-ON-SILICON components have achieved wide deployment in the field of integrated optics, due to their low propagation losses and low polarization dependences. The need for active components, which could compensate for the losses introduced in planar optical circuits, has prompted significant research efforts toward the realization of erbium-doped waveguide amplifiers (EDWAs) [1]-[5]. A few techniques have been proposed for erbium incorporation in optical waveguides, with various degrees of success in terms of net gain per unit length. For wavelength division multiplexing (WDM) network applications, however, high levels of net gain need to be combined with a broad amplification spectrum, which is a key issue for increasing the overall capacity of the system.

For erbium-doped fiber amplifiers (EDFAs), the effects of glass composition on gain spectrum have been extensively investigated. The most common composition is aluminum and germanium codoped silicate glass, which results in a fullwidth at half-maximum broadness (FWHM) of 50-60 nm [6]. Telluride- and fluoride-based fibers have also been proposed, which increase the available spectrum to 70-80 $\mathrm{nm}$ [7]-[9]. A number of techniques based on Bragg gratings [10] and

Manuscript received May 24, 2006; revised December 11, 2006. This work was supported by the Engineering and Physical Sciences Research Council and by Nortel Networks.

A. Laliotis is with the Department of Physics, Imperial College London, SW7 2AZ London, U.K. (e-mail: athanasios.laliotis@imperial.ac.uk).

E. M. Yeatman is with the Optical and Semiconductor Devices Group, Department of Electrical and Electronic Engineering, Imperial College London, SW7 2BT London, U.K. (e-mail: e.yeatman@imperial.ac.uk).

Digital Object Identifier 10.1109/JLT.2007.895561 optical filters [11] are also used to achieve spectral flatness and gain equalization across the channels in the available spectrum.

The high erbium concentration required for EDWAs, as well as the need for integration with existing passive components, significantly reduces the flexibility of dopants and glass types that can be used for this purpose. Moreover, space limitations in planar optical circuits make equalization techniques more difficult to apply than in the fiber case. Efficient ways of increasing the EDWA bandwidth while maintaining high levels of erbium inversion are therefore required.

It is known that phosphorus codoping increases the amount of erbium that can be incorporated in the glass network before precipitation occurs [12]-[14]. For this reason, the highest levels of gain to date have been reported for phosphate-glassbased waveguides [1], [4], [5]. Large amounts of phosphorus doping were also required to achieve net gain levels of about $1 \mathrm{~dB} / \mathrm{cm}$ in waveguide amplifiers based on the sol-gel glass fabrication method [15]. In all the above devices, however, the erbium emission spectrum is below $20 \mathrm{~nm}$ FWHM, in contrast to $60 \mathrm{~nm}$ typically displayed for EDFAs [5].

In this paper, we investigate the effects of phosphorus in the amplifier spectrum and fluorescence decay characteristics. We show that a mere reduction in the levels of phosphorus doping does not produce beneficial effects for the amplifier's spectral performance. Such effects were only achieved for sol-gel aluminosilicate amplifiers. Subsequently, we show that such amplifiers demonstrate good guiding properties and relative gain levels of $\approx 1.5 \mathrm{~dB} / \mathrm{cm}$. However, while these results represent an improvement compared with previous reports on similar compositions [16]-[18], positive net gain has not yet been achieved.

The improved performance was attributed to optimization of the processing conditions, which was carried out for sol-gel waveguide amplifiers. In particular, we focus on minimizing the device exposure to high temperatures, which have been consistently shown in the past to induce erbium clustering [19], [20]. The experimental techniques developed for this purpose are described in detail in this paper.

A novel multilayered (laminated) device is then investigated as a way to combine the spectral characteristics of different host glass compositions and, in particular, to achieve positive net gain as well as spectral broadening. The sol-gel method is particularly well suited for fabricating such laminated structures. Experimental measurements on amplifiers consisting of alternating layers of aluminophosphosilicate and aluminosilicate compositions are presented, and it is shown that spectral benefits that cannot be achieved by the use of single core compositions are demonstrated for this device. 
TABLE I

Glass Host Compositions OF SOL-Gel CORE LAYERS

\begin{tabular}{|c|c|c|c|c|c|}
\hline Sample & \multicolumn{5}{|c|}{ Composition (molar ratio \%) } \\
\hline & $\mathbf{S i O}_{\mathbf{2}}$ & $\mathbf{P}_{\mathbf{2}} \mathbf{O}_{\mathbf{5}}$ & $\mathbf{A l}_{\mathbf{2}} \mathbf{O}_{\mathbf{3}}$ & $\mathbf{E r}_{\mathbf{2}} \mathbf{O}_{\mathbf{3}}$ & $\mathbf{Y b}_{\mathbf{2}} \mathbf{O}_{\mathbf{3}}$ \\
\hline $\boldsymbol{T H 1}$ & 97 & 0 & 2.5 & 0.25 & 0.25 \\
\hline $\boldsymbol{T H} \mathbf{2}$ & 94.5 & 2.5 & 2.5 & 0.25 & 0.25 \\
\hline $\boldsymbol{T H} \mathbf{3}$ & 92 & 5 & 2.5 & 0.25 & 0.25 \\
\hline $\boldsymbol{T H 4}$ & 87 & 10 & 2.5 & 0.25 & 0.25 \\
\hline
\end{tabular}

Numerical analysis is also performed and is shown to be consistent with the experimental results. It is demonstrated that lamination of different compositions can lead to an increase of $20 \mathrm{~nm}$ in the available gain spectrum, compared with previously reported devices, while still maintaining low noise figures.

\section{DeVICE FABRICATION}

One of the main advantages of sol-gel is the fact that various codopants can be easily introduced in the silica network. This feature can be exploited to successfully fabricate EDWAs, as has been demonstrated in the past for aluminophosphosilicate glass host compositions. Evidence of sol-gel metastability [19] suggests that further compositions may be investigated.

The buffer layer for the waveguides under investigation was a $14-\mu$ m-thick thermally grown oxide on a (100) $\mathrm{Si}$ wafer. The active core layer was subsequently deposited by spin coating deposition of sol-gel films. In order to avoid stressinduced cracking, the thickness of each individual layer was $0.1-0.2 \mu \mathrm{m}$, and each deposition was followed by rapid thermal annealing (RTA) at an appropriate temperature, depending on the glass composition [21]. The RTA step was optimized in order to minimize the maximum temperature exposure while maintaining a stress-free film. The process was iteratively followed to achieve a total of 4- $\mu \mathrm{m}$ core layer thickness for each composition. Recently, aluminophosphosilicate sol-gel amplifiers have been reported in which the core can be deposited by a single step [22]. However, a high consolidation temperature $\left(1150^{\circ} \mathrm{C}\right)$ was needed, and net gain has yet to be achieved by this method.

The glass host compositions under investigation are given in Table I, whereas the critical RTA temperature required for thick film deposition is given in Fig. 1. It has been shown, by means of X-ray diffraction measurements, that temperatures above $1000{ }^{\circ} \mathrm{C}$ tend to induce crystallinity centers in erbiumdoped films [23]. For this reason, waveguide amplifiers were not fabricated from TH2 composition. Instead, a laminated core was deposited, consisting of alternating $0.2-\mu \mathrm{m}$ layers of compositions $\mathrm{TH} 1$ and $\mathrm{TH} 4$, giving an average composition whose properties could not be easily reproduced by varying the phosphorus content of a single layer composition. A schematic diagram of this device is shown in Fig. 2.

Subsequent to core deposition, a consolidation step is required to eliminate the porosity of the film. Various studies have revealed that prolonged exposure of erbium-doped films to high temperatures greatly reduces the levels of erbium homogeneity

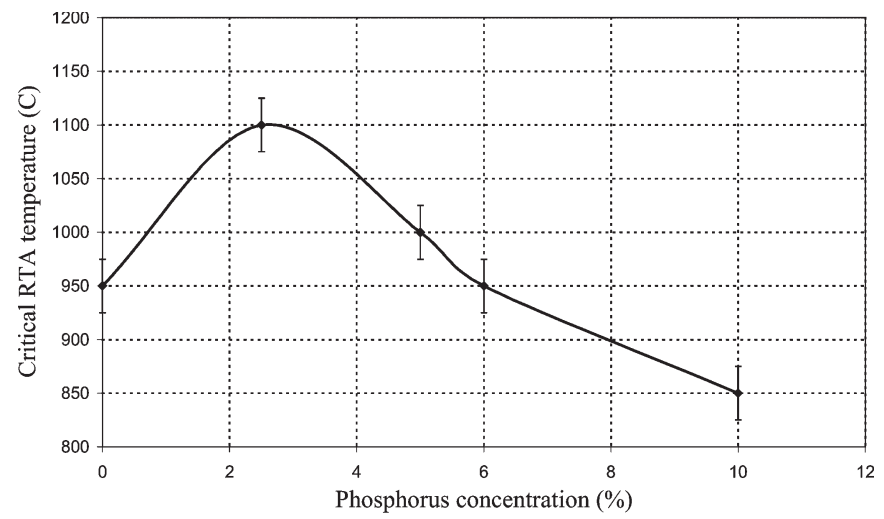

Fig. 1. Dependence of critical RTA temperature on phosphorus doping concentration.

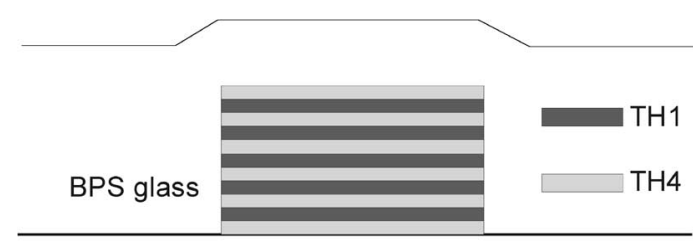

$\mathrm{SiO} 2$

Fig. 2. Schematic diagram of laminated amplifier.

[19], [20]. To determine the appropriate conditions required for this step, the following experimental process was followed. First, single layer films of $\approx 120 \mathrm{~nm}$ thickness were spun on silicon substrates for TH1 composition. This composition was used as a reference, since phosphorus-doped glasses have been shown to have lower melting and reflow temperatures [24]. After spin coating, the silicon wafers were cleaved into pieces of a size appropriate for ellipsometry measurements. The various pieces were then consolidated at temperatures from $900{ }^{\circ} \mathrm{C}$ to $1050{ }^{\circ} \mathrm{C}$ and for times ranging between $5 \mathrm{~min}$ and several hours. The ramping time of the furnace was approximately $1 \mathrm{~h}$. Film consolidation was performed in a neutral argon atmosphere with a $61 / \mathrm{min}$ argon flux.

The film thickness and refractive index were determined for each sample using ellipsometry at $632.8-\mathrm{nm}$ wavelength. By measuring in wet and dry atmospheres, such that the pores are filled with water or air, respectively, film porosity can be calculated using the Lorentz-Lorenz relation [25]-[27], i.e.,

$$
\frac{\left(n_{f}^{2}-1\right)}{\left(n_{f}^{2}+2\right)}=\left(1-v_{p}\right) \frac{\left(n_{s}^{2}-1\right)}{\left(n_{s}^{2}+2\right)}+v_{p} \frac{\left(n_{p}^{2}-1\right)}{\left(n_{p}^{2}+2\right)}
$$

where $n_{f}, n_{s}$, and $n_{p}$ are the film, pore, and skeleton indexes, respectively, and $v_{p}$ is the fractional porosity. The film refractive index and thickness reach a steady-state value as consolidation times increase, and porosity is gradually eliminated [27]. After this point has been reached, excess annealing can only have detrimental effects by further inducing erbium clustering [19]. We therefore define optimum consolidation times as the minimum required to achieve negligible porosity and a constant film refractive index. These are shown in Fig. 3 as a function of temperature. The consolidation conditions followed for all 


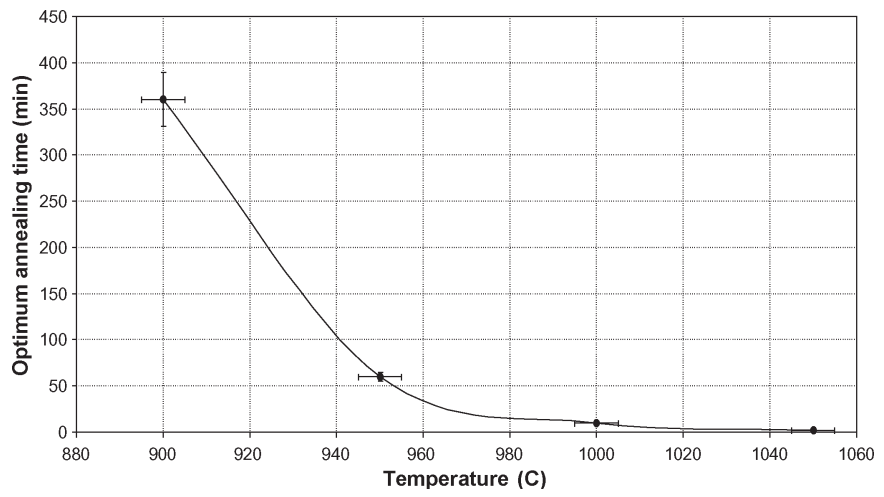

Fig. 3. Optimum annealing time versus temperature for aluminosilicate glass.
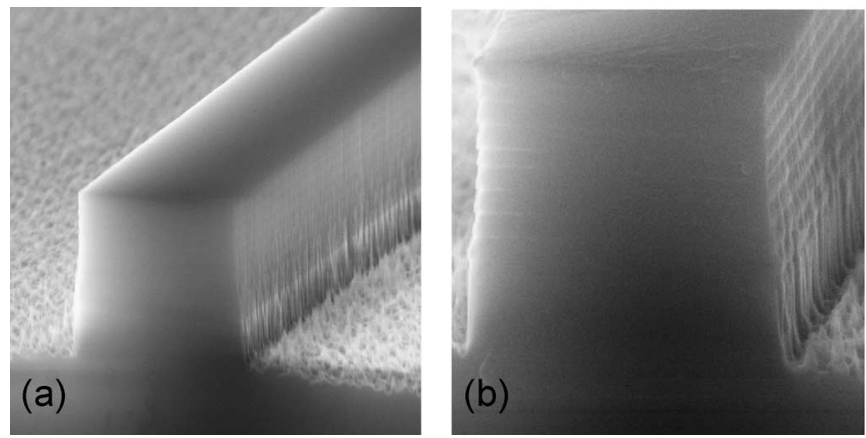

Fig. 4. SEM pictures of laminated amplifier. (a) Waveguide cross section prior to cladding deposition. (b) After a high-frequency etching step to enhance the contrast between individual layers, which are clearly visible.

four samples were $1000{ }^{\circ} \mathrm{C} / 15 \mathrm{~min}$. These results indicate that significant reduction from previously used consolidation times is possible.

The patterning of the core layer was done by dry etching in a Plasma $80 \mathrm{Lab}$, Oxford Instruments reactive ion etcher. Due to the low etch rates of the multicomponent glass and the small feature size (3-6 $\mu \mathrm{m}$ wide waveguides), the mask used was a combination of a $\mathrm{Cr}$ layer $(2000 \AA)$ and a $200{ }^{\circ} \mathrm{C}$ hardbaked photoresist. To avoid reflow of the resist, patterning of the hard-baked layer was done by $\mathrm{O}_{2}$ plasma etching rather than by photolithographic development. An SEM image of the cross section of the laminated amplifier core is shown in Fig. 4. Finally, a $12-\mu \mathrm{m}$-thick cladding layer of borophosphate silicate glass, with refractive index to match that of the thermal oxide, was deposited by plasma enhanced chemical vapor deposition (PECVD).

\section{Single Composition Amplifiers}

Aluminosilicate amplifiers displayed excellent guiding properties. The refractive index of the core was measured to be 1.48 at $632.8-\mathrm{nm}$ wavelength. The index difference between core and cladding is approximately $1.5 \%$, suggesting a good confinement of the fundamental amplifier mode in the waveguide core. The core cross sections of the waveguides reported here are all $4 \times 4 \mu \mathrm{m}$, but similar results were observed for 3-, 5-, and $6-\mu \mathrm{m}$-wide cores (all of $4-\mu \mathrm{m}$ height). The propagation (background) losses were measured via the cutback method to

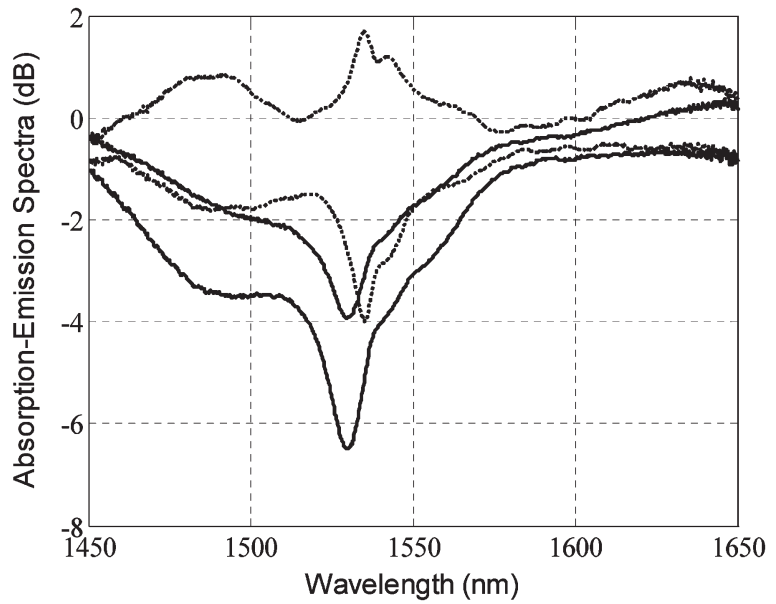

Fig. 5. Absorption and maximum emission (170-mW pump power at $980 \mathrm{~nm})$ for (solid lines) TH1 and (dotted lines) TH4 amplifiers.

be on the order of $0.3 \mathrm{~dB} / \mathrm{cm}$, whereas the coupling losses to single-mode fiber are estimated to be $0.1-0.2 \mathrm{~dB} /$ facet.

The aluminophosphosilicate amplifier cores displayed significantly lower refractive indexes, which is most probably due to the different sol preparation route followed for these compositions. The index difference was $0.3 \%$ for compositions with $5 \% \mathrm{P}_{2} \mathrm{O}_{5}$ content and $0.7 \%$ for compositions with $10 \%$ $\mathrm{P}_{2} \mathrm{O}_{5}$ doping. In this case, the 14- $\mu$ m-thick buffer layer was not enough to prevent optical power leaking to the high index silicon substrate, and the losses measured for this composition were above $5 \mathrm{~dB} / \mathrm{cm}$. Moreover, the losses were linearly increasing with wavelength, suggesting a nonscattering loss mechanism.

Measurements of the amplified spontaneous emission (ASE) spectra were possible for all compositions. Moreover, previously fabricated amplifiers of TH4 composition [19] on 19- $\mu \mathrm{m}$ thick thermal oxide buffer layers, whose background losses were lower than $0.2 \mathrm{~dB} / \mathrm{cm}$, allow us to make a comparison between aluminosilicate and aluminophosphosilicate amplifiers. In Fig. 5, we can see the absorption spectrum of the two compositions, measured with an erbium broadband source and a spectrum analyzer, for 2-cm-long amplifiers. In the same graph, the maximum erbium emission can be seen for pump power levels as high as $180 \mathrm{~mW}$.

In order to make an estimate of the absorption cross sections of the two compositions, one can rely on the evolution equation for signal power [6], i.e.,

$$
\frac{d P_{s}}{d z}=P_{s} \int_{-\infty}^{+\infty} \int_{-\infty}^{+\infty}\left\{\sigma_{e} N_{2}-\sigma_{a} N_{1}\right\} \rho(x, y)|\psi(x, y)|^{2} d x d y
$$

where $\sigma_{a, e}$ are the absorption and emission cross sections, $\psi$ is the normalized mode profile, and $\rho$ is the erbium doping profile. The population densities of the ground and metastable levels are denoted by $N_{1}$ and $N_{2}$, respectively. In our case, the erbium doping is constant and confined in the waveguide core. In the absence of the pump, the erbium populations $N_{1}$ and 
$N_{2}$ are constant over the entire amplifier length, and (2) can be integrated to give

$$
G=e^{\Gamma \sigma_{a}\left(\eta N_{2}-N_{1}\right) L}
$$

where $G$ is the maximum erbium absorption, $L$ is the total amplifier length, $\eta$ is the degeneracy factor (the ratio of emission and absorption cross sections at the specific wavelength), and $\Gamma$ is a confinement factor denoting the fraction of the total mode power that is in the core.

With no pump, one can assume that all ions are at the ground level. As aforementioned, the amplifier length was $2 \mathrm{~cm}$, and the erbium concentration was about $1.1 \times 10^{26}$ ions $/ \mathrm{m}^{3}$. From Fig. 5, one can conclude that the peak erbium-induced absorption is 5.2 and $3.25 \mathrm{~dB}$ for TH1 and TH 4 compositions, respectively. The confinement factor $\Gamma$ was calculated to be 0.92 for TH 1 and 0.83 for TH 4 composition by means of the Method of Lines [31], which is described in more detail in Section IV. Using (3), we can calculate that the peak absorption cross section for the aluminosilicate composition (TH1) is $\sigma_{a}=6.1 \times 10^{-25} \mathrm{~m}^{2}$, whereas for the aluminophosphosilicate composition (TH4), $\sigma_{a}=4.2 \times 10^{-25} \mathrm{~m}^{2}$. In the case of aluminosilicate hosts, values typically reported in literature [6] estimate the absorption cross section of erbium to be on the order of $6 \times 10^{-25} \mathrm{~m}^{2}$, which is in good agreement with our measurements. Phosphate glass amplifiers are reported to have somewhat lower absorption cross sections of $\approx 5.2 \times 10^{-25} \mathrm{~m}^{2}$ [5], which is a value still somewhat higher than that we obtained for sol-gel amplifiers with high phosphorus content. Though the different final compositions and fabrication routes followed in the two cases could account for the observed difference in the cross sections, it is possible that uncertainty in the measurement of the total erbium concentration could (at least partly) be responsible. An additional likely source of error is the use of refractive indexes measured at $633 \mathrm{~nm}$ rather than $1550 \mathrm{~nm}$.

It should also be noted that the value of the total erbium concentration for both compositions was inferred from the stoichiometry of the initial solutions. Energy dispersive $\mathrm{X}$-ray measurements performed on the amplifier cross sections revealed the presence of rare earths in the waveguide cores; however, quantitative comparisons could not be made between the two waveguides with any precision.

Estimation of the emission cross section requires knowledge of the degeneracy factor. Even though this varies significantly according to glass host, it is reported that for aluminum and phosphorus codoped compositions, $\eta$ is close to unity [6] at the maximum absorption wavelength. By making this assumption, one can also calculate the levels of erbium inversion for each composition.

By making use of (3) and making straightforward calculations, one can derive the following equation for the population of the erbium metastable level:

$$
N_{2}=\frac{1}{\eta+1}\left[1-\left(\frac{G_{e}(\text { in } \mathrm{dB})}{G_{a}(\text { in } \mathrm{dB})}\right)\right] N_{\text {tot }}
$$

where $G_{a}$ and $G_{e}$ are the gain levels with and without pump, respectively, and $N_{\text {tot }}$ is the total erbium doping. Using (3),

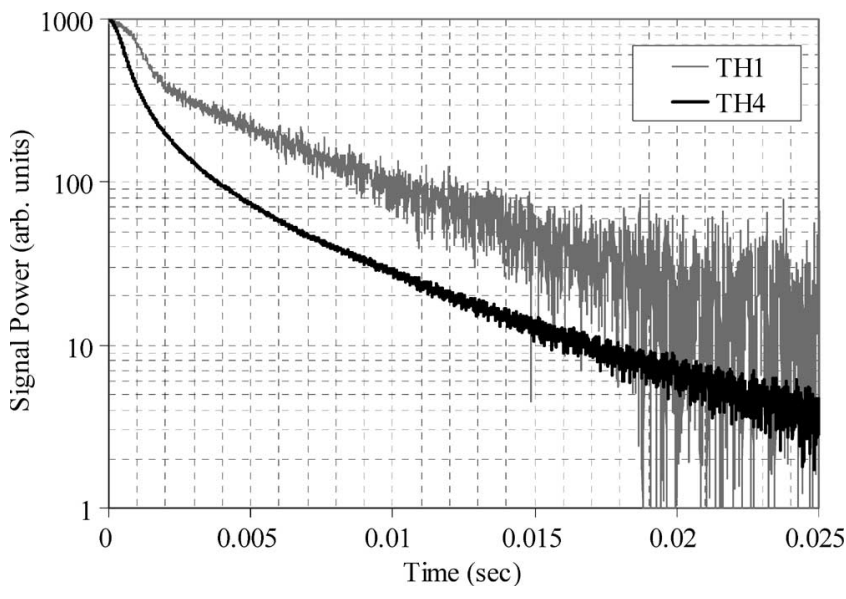

Fig. 6. Fluorescence decay of (gray line) TH1 and (black line) TH4 compositions.

we can calculate that TH1 composition has $25 \%$ erbium inversion, whereas TH4 has approximately $83 \%$.

It is possible that phosphorus codoping reduces the erbium cross sections significantly, thus reducing the maximum achievable gain of a waveguide amplifier. However, it is clear from Fig. 5 that the levels of erbium inversion are not sufficient in this case for net gain to be reached. This is probably due to low levels of homogeneity of the erbium ion environment. A potential increase of the aluminum content of aluminosilicate compositions to 5\%-7.5\% could, however, assist in the dissolution of clusters [13], [28] and result in similar homogeneity levels to those in TH4 composition. In this case, the levels of net gain that could be expected are on the order of $1.5-2 \mathrm{~dB} / \mathrm{cm}$, which are significantly higher than those so far observed for aluminophosphosilicate amplifiers.

For a close examination of erbium homogeneity, the fluorescence decay of the two compositions was measured and is shown in Fig. 6. These measurements were performed by mechanically modulating the pump laser signal through a chopper. The maximum available pump power through this configuration was about $100 \mathrm{~mW}$ due to the losses of the lens-chopper-lens system. The switch-off time for the pump in these measurements, which is calculated to be less than $0.5 \mathrm{~ms}$, sets a limit to the phenomena that can be measured by this experiment. However, this limit is small enough to ensure accurate determination of the metastable level lifetime, as well as cooperative upconversion mechanisms. To ensure more uniform pumping through the waveguides, we used $1-\mathrm{cm}$ long guides of TH1 and TH4 compositions. The timedependent fluorescence of the amplifier was measured by a $\mathrm{Ge}$ detector, after being filtered through a high-pass filter to reduce noise from the residual pump.

Contrary to what was expected, a slower decay was observed for the aluminosilicate amplifiers. However, this is most probably due to the fact that the large fraction of clustered ions is not sufficiently inverted for their fluorescence to be observed. For this purpose, the levels of pump power required are on the order of a few watts [29]. Furthermore, phenomena related to erbium clustering might be considerably faster than can be measured in this experiment, which is limited by both pump switch-off 


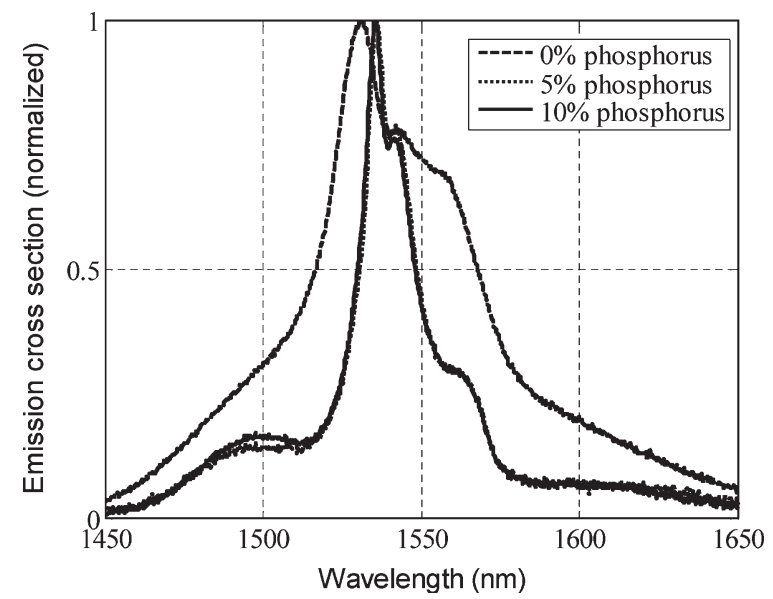

Fig. 7. Normalized emission cross sections of the three single core compositions $\mathrm{TH} 1, \mathrm{TH} 3$, and $\mathrm{TH} 4\left(0 \%, 5 \%\right.$, and $10 \% \mathrm{P}_{2} \mathrm{O}_{5}$, respectively).

time and the bandwidth of the photodetector. The lifetime of the metastable level, however, can be estimated from Fig. 6 to be on the order of $6.5 \mathrm{~ms}$ for TH1 and $8 \mathrm{~ms}$ for the TH4 composition, which is comparable to other values reported in the literature for silicate hosts [6], [15], [30]-[33]. It should be noted that the results of the lifetime measurements have a dependence on the signal-to-noise ratio in the detector, which is a fact that reduces their accuracy significantly. A direct comparison of measurements is therefore difficult, since the experimental error is estimated to be on the order of $2 \mathrm{~ms}$.

The emission spectra of all three single core compositions have been inferred from the ASE spectra, which have been measured in the absence of signal power. In order to minimize the error, which might be caused by ASE absorption and reemission along the waveguide, the spectral dependence of the emission cross sections has been determined for 1-cm-long amplifiers. The normalized emission cross sections of all three compositions are presented in Fig. 7. It is clear that phosphorus doping reduces the breadth of the amplifier's spectrum from 55 to $18 \mathrm{~nm}$ : a reduction that is detrimental for the capacity of WDM networks. Reduced levels of phosphorus doping have negligible effects on the erbium emission cross section. One can therefore conclude that even though phosphorus assists in the dissolution of clusters, as is demonstrated through spectroscopic and fluorescence decay measurements, it reduces both spectral broadness and the peak value of erbium cross sections.

We can conclude that even though a single core composition suggests itself as a way to create amplifiers with new spectral properties, this may not be practically possible. Consequently, we propose here and demonstrate that multilayered devices provide a feasible route to successfully combine the spectral properties and benefits of different host glass compositions.

\section{LAMINATED AMPLIFIERS}

The laminated amplifier that was fabricated consists of alternating individual layers $(0.2 \mu \mathrm{m}$ thick) of aluminosilicate (TH1) and aluminophosphosilicate (TH4) compositions. The total height of the core was $4 \mu \mathrm{m}$, and the width of the waveguides was $6 \mu \mathrm{m}$. The waveguides were patterned on a

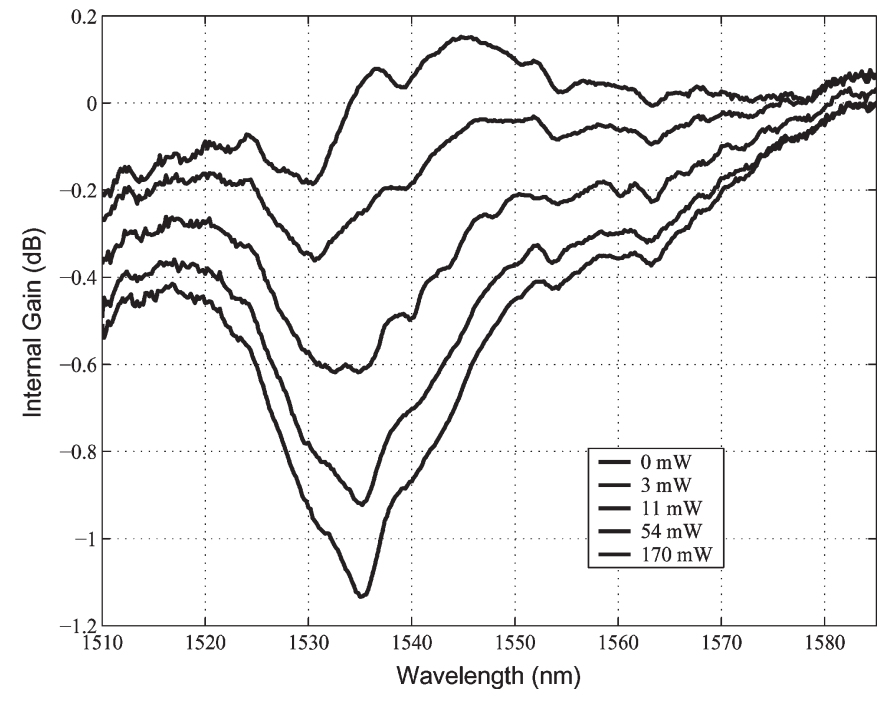

Fig. 8. Internal gain of laminated amplifier for increasing pump powers as shown.

14- $\mu$ m-thick thermal oxide. The high index of the aluminosilicate composition improves the mode confinement, thus significantly improving the guiding properties of the device. The confinement factor in this case is calculated to be 0.89 . Cutback measurements on laminated amplifiers showed propagation losses of approximately $1 \mathrm{~dB} / \mathrm{cm}$. Most of this is due, as in the case of aluminophosphosilicate amplifiers, to power leakage into the silicon substrate. Coupling losses to single-mode fiber are estimated to be on the order of $0.3 \mathrm{~dB} /$ facet. The amplifier internal gain for different pump powers is shown in Fig. 8 for a 1-cm-long waveguide. Evidence of erbium ion inversion is visible in this case, suggesting that net gain could be achieved with better control of the cladding and buffer properties.

The emission cross section of erbium ions is usually characteristic of the glass host composition. In the case of the laminated amplifier, where layers of different compositions are used, one can infer an effective emission cross section from the ASE spectrum of the amplifier, as in the case of single composition cores. In Fig. 9, we can see the emission cross sections of the laminated amplifier as well as that of the aluminophosphosilicate amplifier. An increase of 4-5 nm in the available spectrum is observed, which could not be achieved by means of a single core composition, as previously demonstrated. A lower index cladding and buffer would help to further improve the performance and give better insight into the characteristics of this device.

The above results demonstrate that layered structures can, in principle, successfully combine the spectral characteristics of different compositions. It is, however, evident that higher inversion is required to achieve useful net gain. This could be achieved by improving the performance of aluminosilicate compositions and by further optimizing the waveguide design in order to minimize the background losses, which, in this case, are high. This can be achieved by increasing the ratio of aluminum to rare earth ions in the composition, which has been known to improve the performance of $\mathrm{Nd}$-doped waveguide amplifiers [13] but has also been theoretically predicted [28]. It should also be pointed out that the actual ratios of the 


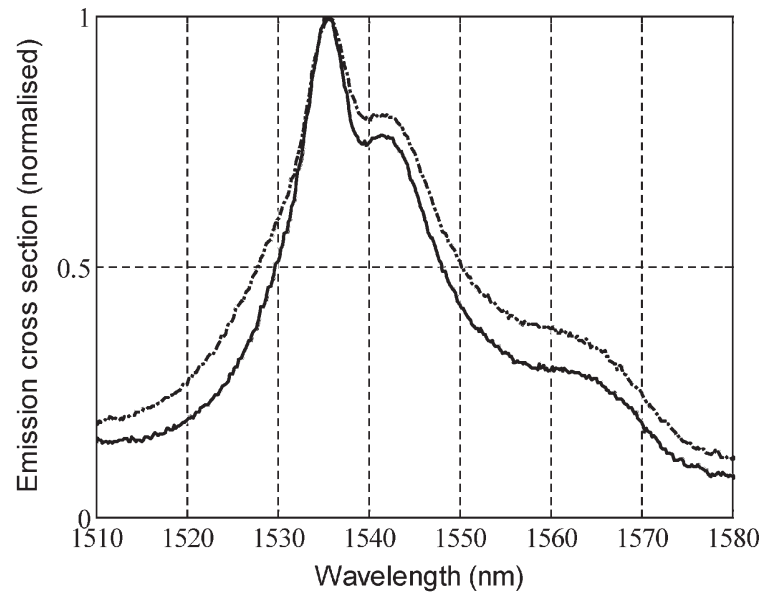

Fig. 9. Normalized emission cross sections of (dashed-dotted line) laminated amplifier and (solid line) aluminophosphosilicate amplifier (TH3).

individual layers of a laminated amplifier could act as an additional degree of freedom for tailoring the final amplifier characteristics. In our case, an increase of TH4 layer thickness would result in an amplifier with higher gain but smaller spectral width, and vice versa.

We have further investigated the advantages of the laminated structure by numerical modeling. The method used is the Method of Lines, which has been applied before for the modeling of bent [31] and straight active waveguides [19]. The details of the method have been reported for various configurations [31], [32]. Here, we have assumed copropagating pump and signal beams, with pumping at $980-\mathrm{nm}$ wavelength. The basic principle consists of the discrimination of the amplifier lateral direction ( $x$-axis) in lines of uniform refractive index. The direction of propagation is divided into independent steps whose gain is calculated by means of the rate equations, which, in this case, assume erbium to be a four-level system including ytterbium sensitization, as given by [31]

$$
\begin{aligned}
\frac{d N_{1}}{d t}= & W_{12} N_{2}-W_{21} N_{1}+W_{13} N_{3}-W_{31} N_{1} \\
& +\frac{N_{2}}{\tau_{2}}+\frac{N_{3}}{\tau_{3}}+K_{2} N_{2}^{2}+K_{3} N_{3}^{2}-C_{\mathrm{up}} N_{1} N_{6} \\
\frac{d N_{2}}{d t}= & W_{12} N_{1}-W_{21} N_{2}-\frac{N_{2}}{\tau_{21}}+\frac{N_{3}}{\tau_{32}}-2 K_{2} N_{2}^{2} \\
\frac{d N_{3}}{d t}= & W_{13} N_{1}-W_{31} N_{3}-\frac{N_{3}}{\tau_{32}}-\frac{N_{3}}{\tau_{31}}+\frac{N_{4}}{\tau_{41}} \\
& -2 K_{3} N_{3}^{2}-C_{\mathrm{up}} N_{1} N_{6} \\
\frac{d N_{4}}{d t}= & -\frac{N_{4}}{\tau_{41}}+K_{2} N_{2}^{2}+K_{3} N_{3}^{2} \\
\frac{d N_{5}}{d t}= & W_{65} N_{6}-W_{56} N_{5}+\frac{N_{6}}{\tau_{65}}+C_{\mathrm{up}} N_{1} N_{6} \\
\frac{d N_{6}}{d t}= & W_{56} N_{5}-W_{65} N_{6}-\frac{N_{6}}{\tau_{65}}-C_{\mathrm{up}} N_{1} N_{6} .
\end{aligned}
$$

The constants $W_{i j}$ and $\tau_{i j}$ represent the transition rates and lifetimes, respectively, between levels $i$ and $j$, whereas $K_{2}$ and $K_{3}$ are the cooperative upconversion constants from levels
TABLE II

COEFFICIENTS USED FOR THE NUMERICAL MODELING

\begin{tabular}{|l|}
\hline$\lambda_{\mathrm{s}}=1535 \mathrm{~nm}$ \\
\hline$\lambda_{\mathrm{p}}=980 \mathrm{~nm}$ \\
\hline $\mathrm{t}_{31}=2 \times 10^{-5} \mathrm{sec}$ \\
\hline$\tau_{2}=8 \mathrm{msec}(\mathrm{TH} 4)$ \\
\hline$\tau_{2}=6.5 \mathrm{msec}(\mathrm{TH} 1)$ \\
\hline$\tau_{41}=1 \times 10^{-9} \mathrm{sec}$ \\
\hline$\tau_{32}=1 \times 10^{-5} \mathrm{sec}$ \\
\hline$\tau_{65}=1 \mathrm{msec}$ \\
\hline $\mathrm{C}_{\mathrm{up}}=2.3 \times 10^{-4} \mu \mathrm{m}^{3} / \mathrm{sec}^{-}$ \\
\hline $\mathrm{K}_{2}=\mathrm{K}_{3}=1.8 \times 10^{-5} \mu \mathrm{m}^{3} / \mathrm{sec}$ \\
\hline$\sigma_{12}=\sigma_{21}=4.2 \times 10^{-13} \mu \mathrm{m}^{2}(\mathrm{TH} 4)$ \\
\hline$\sigma_{12}=\sigma_{21}=6.1 \times 10^{-13} \mu \mathrm{m}^{2}(\mathrm{TH} 1)$ \\
\hline$\sigma_{13}=\sigma_{31}=2.58 \times 10^{-13} \mu \mathrm{m}^{2}$ \\
\hline$\sigma_{56}=\sigma_{65}=9 \times 10^{-13} \mu \mathrm{m}^{2}$ \\
\hline
\end{tabular}

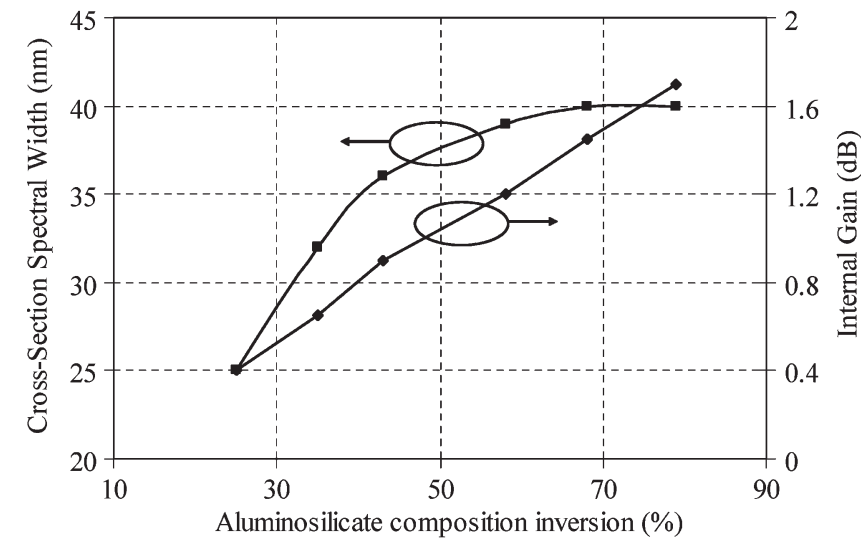

Fig. 10. ASE spectral width and internal gain of a laminated amplifier as a function of the percentage of erbium ions in the metastable level in the aluminosilicate layers.

$\mathrm{I}_{13 / 2}$ and $\mathrm{I}_{9 / 2}$. The ytterbium sensitization is described through levels 5 and 6, and the $C_{\text {up }}$ is the erbium-ytterbium interaction constant. Similarly, $\sigma_{i j}$ is the absorption cross section for the transition from level $i$ to $j$. The cross sections of the metastable level were $6.1 \times 10^{-25} \mathrm{~m}^{2}$ for the aluminosilicate and $4.2 \times$ $10^{-25} \mathrm{~m}^{2}$ for the aluminophosphosilicate composition, as reported above. The calculation is followed until the final signal output is calculated at the amplifier's end. The parameters used for our simulations are shown in Table II [19], [29]-[36].

It was experimentally demonstrated that for a ratio $r=5$ of aluminum to rare earth concentration, the level of erbium inversion observed was $25 \%$ for aluminosilicate composition TH1. An increase in the levels of aluminum doping is expected to increase the levels of homogeneity and, therefore, of erbium inversion. The expected performance (spectral width of the effective emission cross section and internal gain) of a $1-\mathrm{cm}-$ long laminated amplifier, as a function of the inversion in the aluminosilicate layers, is shown in Fig. 10. The parameters governing erbium populations for the aluminophosphosilicate layers are assumed constant, as reported in [19]. The levels of inversion of aluminosilicate levels are varied by means of the erbium upconversion constant $\left(K_{2}\right)$. 


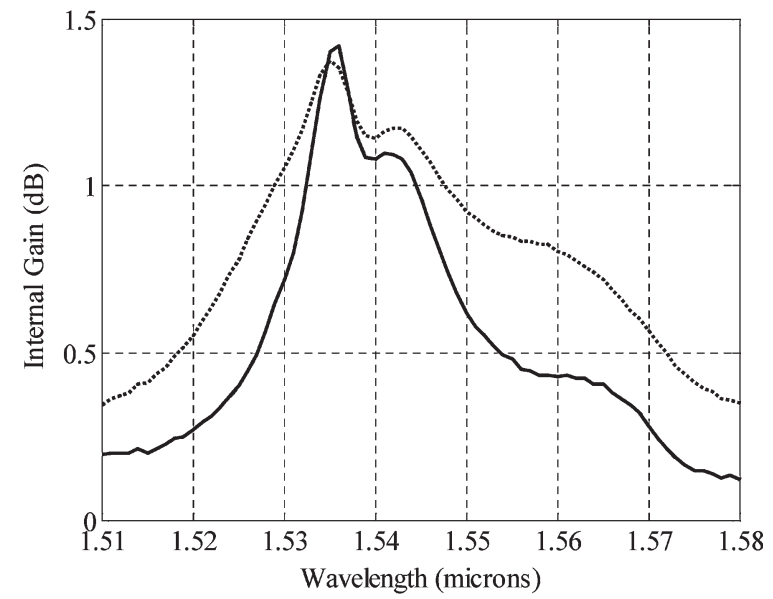

Fig. 11. Modeled internal gain of (solid line) TH4 composition and (dotted line) laminated amplifier.

Fig. 10 shows that for erbium inversion of $25 \%$, the internal gain is $0.4 \mathrm{~dB}$, and the spectral ASE width is $25 \mathrm{~nm}$, which is consistent with the experimentally measured values. Moreover, we can see that internal gain levels of approximately $1.4 \mathrm{~dB} / \mathrm{cm}$ are expected with erbium inversion of $65 \%$. It is therefore clear that the laminated amplifier can achieve similar gain levels to aluminophosphosilicate amplifiers, even when the level of homogeneity in aluminosilicate layers is low. This is a considerable advantage considering that high inversion levels are difficult to achieve in this glass host [3], [16]-[18]. In this case, the spectral width of the laminated amplifier is $37 \mathrm{~nm}$, as opposed to only $18 \mathrm{~nm}$ for the TH4 composition. The spectral advantages of laminated devices are more clearly demonstrated in Fig. 11, where the spectral gains of the laminated and TH4 amplifiers are shown. It should be noted that the cost for higher amplification bandwidth in this case is an increase in the device noise figure. Calculations based on the spontaneous emission factor (high gain regime) reveal that the noise figure for a single core amplifier of TH4 composition is $3.8 \mathrm{~dB}$, in contrast to $4.6 \mathrm{~dB}$ for a laminated amplifier. This degradation, however, could be considered a modest price to pay, given that a single core aluminosilicate composition with $65 \%$ inversion would display a noise figure of $6.4 \mathrm{~dB}$.

\section{CONCLUSION}

A comparative study of a range of sol-gel amplifier compositions is presented in this paper. Spectroscopic characterization and fluorescence decay measurements reveal that even though phosphorus assists in the dissolution of erbium clusters, thus increasing the levels of erbium homogeneity, it significantly reduces the absorption cross section of rare earth ions and their available emission spectrum. To overcome this problem, a novel device consisting of multiple layers of aluminosilicate and aluminophosphosilicate glass hosts was fabricated. It is shown that such a device can achieve spectral characteristics that are difficult to obtain in a single composition core. Numerical simulations show that the available gain spectrum for WDM networks of a laminated amplifier is significantly increased in comparison with phosphorus codoped amplifiers, while maintaining equal levels of gain. The numerical results are further supported by experimental measurements, and good agreement is established.

\section{ACKNOWLEDGMENT}

The authors would like to thank Dr. H. Ou of the Technical University of Denmark for the PECVD cladding deposition of our samples and R. R. A. Syms and M. M. Ahmad of Imperial College for useful discussions and assistance with sample fabrication.

\section{REFERENCES}

[1] D. Barbier, P. Bruno, C. Cassagnettes, M. Trouillon, R. L. Hyde, A. Kevorkian, and J. M. P. Deleveauxl, "Net gain of $27 \mathrm{~dB}$ with 8.6-cm-long $\mathrm{Er} / \mathrm{Yb}$ doped glass planar amplifier," in OFC Tech. Dig., 1998, pp. 45-48.

[2] P. Camy, J. E. Román, F. W. Willems, M. Hempstead, J. S. Wilkinson, J. C. van der Plaats, A. M. J. Koonen, A. Béguin, C. Prel, and C. Lerminiaux, "Ion-exchanged planar lossless splitter at $1.5 \mu \mathrm{m}, "$ Electron. Lett., vol. 32, no. 4, pp. 321-323, Feb. 1996.

[3] J. Hübner, S. Guldberg-Kjaer, M. Dyngaard, Y. Shen, C. L. Thomsen, S. Balslev, C. Jensen, D. Zauner, and T. Feuchter, "Planar Er and Yb doped amplifiers and lasers," Appl. Phys. B, Photophys. Laser Chem., vol. 73, no. 5/6, pp. 435-438, 2001.

[4] F. D. Patel, S. DiCarolis, P. Lum, S. Venkatesh, and J. N. Miller, "A compact high performance optical waveguide amplifier," IEEE Photon. Technol. Lett., vol. 16, no. 12, pp. 2607-2609, Dec. 2004.

[5] Y. C. Yan, A. J. Faber, H. de Waal, P. G. Kik, and A. Polman, "Erbiumdoped phosphate glass waveguide on silicon with $4.1 \mathrm{~dB} / \mathrm{cm}$ gain at 1.535 нm," Appl. Phys. Lett., vol. 71, no. 20, pp. 2922-2924, Nov. 1997.

[6] E. Desurvire, Erbium Doped Fiber Amplifiers: Principles and Applications. New York: Wiley-Interscience, 1994.

[7] H. Ono, M. Yamada, and Y. Ohishi, "Gain-flattened Er-doped fiber amplifier for WDM signal in the 1.57-1.6 $\mu \mathrm{m}$ wavelength region," IEEE Photon. Technol. Lett., vol. 9, no. 5, pp. 596-598, May 1997.

[8] M. Yamada et al., "Gain-flattened tellurite-based EDFA with flat amplification bandwidth of $76 \mathrm{~nm}$," IEEE Photon. Technol. Lett., vol. 10, no. 9, pp. 1244-1246, Sep. 1998.

[9] Y. Ohishi et al., "Gain characteristics of tellurite-based erbium doped fiber amplifiers for $1.5 \mu \mathrm{m}$ broadband amplification," Opt. Lett., vol. 23, no. 4, pp. 274-276, Feb. 1998.

[10] S. K. Liaw et al., "Dynamic power-equalised EDFA module based on strain tuneable Bragg gratings," IEEE Photon. Technol. Lett., vol. 11, no. 7, pp. 797-799, Jul. 1999.

[11] L. R. Chen et al., "Transmission edge filters for power equalisation of EDFA's," IEEE Photon. Technol. Lett., vol. 12, no. 7, pp. 822-824, Jul. 2000.

[12] W. J. Miniscalco, "Erbium-doped glasses for fiber amplifiers at $1500 \mathrm{~nm}$," J. Lightw. Technol., vol. 9, no. 2, pp. 234-250, Feb. 1991.

[13] K. Arai, H. Namikawa, K. Kumata, and T. Honda, "Aluminium or phosphorus co-doping effects on the fluorescence and structural properties of neodymium-doped silica glass," J. Appl. Phys., vol. 59, no. 10, pp. 3430-3436, May 1986.

[14] K. Hattori, T. Kitakawa, M. Oguma, H. Okazaki, and Y. Ohmori, "Optical amplification in $\mathrm{Er}^{+3}$ doped $\mathrm{P}_{2} \mathrm{O}_{5}-\mathrm{SiO}_{2}$ planar waveguides," J. Appl. Phys., vol. 80, no. 9, pp. 5301-5308, Nov. 1996.

[15] W. Huang, R. R. A. Syms, E. M. Yeatman, M. M. Ahmad, T. V. Clap, and S. M. Ojha, "Fiber-device-fiber gain from a sol-gel erbium-doped waveguide amplifier," IEEE Photon. Technol. Lett., vol. 14, no. 7, pp. 959-961, Jul. 2002.

[16] E. M. Yeatman, M. M. Ahmad, O. McCarthy, A. Vannucci, P. Gastaldo, D. Barbier, D. Mongardien, and C. Moronvalle, "Optical gain in Er-doped $\mathrm{SiO}_{2}-\mathrm{TiO}_{2}$ waveguides fabricated by the sol-gel technique," Opt. Commun., vol. 164, no. 1-3, pp. 19-25, Jun. 1999.

[17] W. Huang and R. R. A. Syms, "Sol-gel silica on silicon buried channel EDWA's," J. Lightw. Technol., vol. 21, no. 5, pp. 1339-1349, May 2003.

[18] K. Solehmainen, M. Kapulainen, P. Heimala, and K. Polamo, "Erbium doped waveguides fabricated with atomic layer deposition method," IEEE Photon. Technol. Lett., vol. 16, no. 1, pp. 194-196, Jan. 2004.

[19] A. Laliotis, E. M. Yeatman, M. M. Ahmad, and W. Huang, "Molecular homogeneity in erbium-doped sol-gel waveguide amplifiers," IEEE $J$. Quantum Electron., vol. 40, no. 6, pp. 805-814, Jun. 2004. 
[20] M. W. Sckerl, S. Guldberg-Kjaer, M. Rysholt Poulsen, P. Shi, and J. Chevallier, "Precipitate coarsening and self organization in erbiumdoped silica," Phys. Rev. B, Condens. Matter, vol. 59, no. 21, pp. $13494-$ 13 497, Jun. 1999.

[21] R. R. A. Syms, A. S. Holmes, W. Huang, V. M. Schneider, and M. Green, "Development of the SC-RTA process for fabrication of sol-gel based silica-on-silicon integrated optic components," J. Sol-Gel Sci. Technol., vol. 13 , no. $1-3$, pp. 509-516, 1998.

[22] R. R. Thomson, H. T. Bookey, H. Ur-Rehman, S. Liu, N. Suyal, and A. K. Kar, "Optically active erbium-doped waveguides fabricated using a single sol-gel deposition technique," J. Lightw. Technol., vol. 23, no. 12, pp. 4249-4256, Dec. 2005.

[23] E. M. Yeatman, M. M. Ahmad, and O. McCarthy, "Sol-gel fabrication of rare-earth photonic components," J. Sol-Gel Sci. Technol., vol. 19, no. 1-3, pp. 231-236, Dec. 2000.

[24] R. R. A. Syms, W. Huang, and V. M. Schneider, "Optimisation of borophosphosilicate glass compositions for silica-on-silicon integrated optical circuits fabricated by the sol-gel process," Electron. Lett., vol. 32, no. 13, pp. 1233-1234, Jun. 1996.

[25] M. Born and E. Wolf, Principles of Optics. London, U.K.: Cambridge Univ. Press, 1997.

[26] M. A. Fardad, E. M. Yeatman, E. J. C. Dawnay, M. Green, and F. Horowitz, "Effects of $\mathrm{H}_{2} \mathrm{O}$ on structure of acid catalysed $\mathrm{SiO}_{2}$ sol-gel films," J. Non-Cryst. Solids, vol. 183, no. 3, pp. 260-267, Apr. 1995.

[27] A. Laliotis and E. M. Yeatman, "Consolidation and homogeneity of erbium doped waveguide amplifiers," presented at the Conf. Lasers and Electro-Optics/Int. Council Quantum Electronics PhAST, San Francisco, CA, May 2004, Paper CTuS2.

[28] J. Laegsgaard, "Dissolution of rare-earth clusters in $\mathrm{SiO}_{2}$ by $\mathrm{Al}$ codoping: A microscopic model," Phys. Rev. B, Condens. Matter, vol. 65, no. 17, pp. 174114-1-174114-10, Apr. 2002.

[29] J. Philipsen, P. Gastaldo, C. Cassagnettes, D. Barbier, A. Kevorkian, and A. Yeniay, "Self-referenced Q-switched pump-probe transmission experiment for the determination of the degree of clustering in Er-doped planar waveguides," in Proc. Opt. Amplifiers and Their Appl., OSA Trends Opt. Photon., 1999, vol. 30, pp. 80-83.

[30] G. Nykolak, P. C. Becker, J. Shmulovitch, Y. H. Wong, D. J. DiGiovanni, and A. J. Bruce, "Concentration dependant ${ }^{4} \mathrm{I}_{13 / 2}$ lifetimes in Er-doped fibers and Er-doped planar waveguides," IEEE Photon. Technol. Lett., vol. 5, no. 9, pp. 1014-1016, Sep. 1993.

[31] W. Huang and R. R. A. Syms, "Analysis of folded erbium-doped planar waveguide amplifiers by the method of lines," J. Lightw. Technol., vol. 17, no. 12, pp. 2658-2664, Dec. 1999.

[32] A. Laliotis, E. M. Yeatman, and S. J. Al-Bader, "Modelling signal and ASE evolution in erbium doped amplifiers by the method of lines," J. Lightw. Technol., vol. 24, no. 3, pp. 1589-1600, Mar. 2006.
[33] F. Di Pasquale and C. W. Pitt, "Improved gain performance characteristics in high concentration $\mathrm{Er} / \mathrm{Yb}$ codoped glass waveguide amplifiers," IEEE J. Quantum Electron., vol. 30, no. 9, pp. 2127-2131, Sep. 1994.

[34] F. Di Pasquale and M. Federighi, "Modelling of uniform and pair induced upconversion mechanisms in high concentration erbium doped silica waveguides,” J. Lightw. Technol., vol. 13, no. 9, pp. 1858-1864, Sep. 1995.

[35] O. Lumholt, T. Rasmussen, and A. Bjarklev, "Modelling of extremely high concentration erbium-doped silica waveguides," Electron. Lett., vol. 29, no. 5, pp. 495-496, Mar. 1993.

[36] C. Strohhofer and A. Polman, "Relationship between concentration in $\mathrm{Er}^{3+}-\mathrm{Yb}^{3+}$ doped waveguide amplifiers," J. Appl. Phys., vol. 90, no. 9, pp. 4314-4320, 2001.

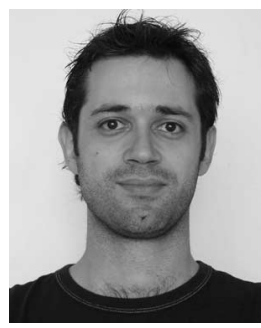

Athanasios Laliotis received the B.Sc. degree in physics from the University of Athens, Athens, Greece, in 1998, the M.S.E. degree in electrical engineering from Princeton University, Princeton, NJ, in 2000, and the Ph.D. degree from Imperial College London, London, U.K., in 2005.

He was a Research Associate with the University of Paris, Paris, France. He is currently with the Department of Physics, Imperial College London. His research interests include integrated optical devices, with emphasis on atom chips for cold atom manipulation.

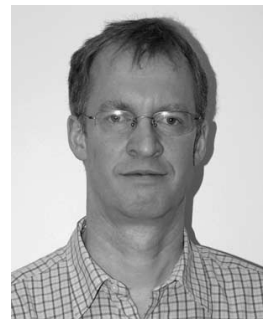

Eric M. Yeatman (M'01-SM'05) received the B.Eng. degree in engineering physics and the M.Sc. degree in physics from Dalhousie University, Halifax, NS, Canada, in 1985 and 1986, respectively, and the Ph.D. degree from Imperial College London, London, U.K., in 1989.

He has been a member of Staff with the Department of Electrical and Electronic Engineering, Imperial College London, since 1989, the Deputy Head of the Optical and Semiconductor Devices Group since 1996, and a Professor of microengineering since 2005. His current research includes micromechanical actuators and generators, microstructures for optical and microwave applications, and integrated optical amplifiers. 